\title{
The Case for Expanding the Midwifery-led Model of Care
}

Michelle Winokur, DrPH, and the AfPA Governmental Affairs Team, Alliance for Patient Access (AfPA)

The Alliance for Patient Access (allianceforpatientaccess.org), founded in 2006, is a national network of physicians dedicated to ensuring patient access to approved therapies and appropriate clinical care. AfPA accomplishes this mission by recruiting, training and mobilizing policy-minded physicians to be effective advocates for patient access. AfPA is organized as a non-profit 501(c)(4) corporation and headed by an independent board of directors. Its physician leadership is supported by policy advocacy management and public affairs consultants. In 2012, AfPA established the Institute for Patient Access (IfPA), a related 501(c) (3) non-profit corporation. In keeping with its mission to promote a better understanding of the benefits of the physician-patient relationship in the provision of quality healthcare, IfPA sponsors policy research and educational programming.

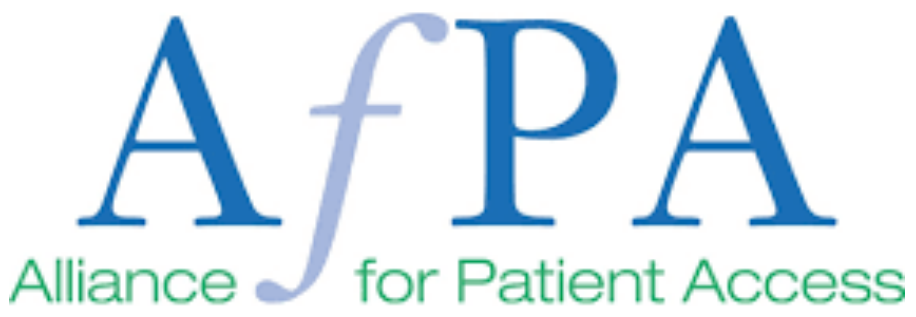

While the novel coronavirus has rocked the country's health and economic systems, we cannot let it overshadow other public health issues that existed prior to it, and that will continue beyond it. One such issue is poor maternal and child health outcomes, the topic of a new Institute for Medicaid Innovation resource report. The report highlights an intriguing potential solution: expanding the midwifery-led model of care.

"While the novel coronavirus has rocked the country's health and economic systems, we cannot let it overshadow other public health issues that existed prior to it, and that will continue beyond it. One such issue is poor maternal and child health outcomes, the topic of a new Institute for Medicaid Innovation resource report. "
"Improving Maternal Health Access, Coverage, and Outcomes in Medicaid" defines midwifery-led continuity of care as care provided when the midwife leads the "planning, organization, and delivery of care given to a woman from initial booking to the postnatal period." It can occur in a variety of practice settings including freestanding or alongside birth centers as well as hospitals and clinics.

In contrast, traditional obstetrical care is usually provided in a hospital setting by a medical doctor, and it typically involved intensive monitoring. While that level of care may be appropriate for high-risk pregnancies, the report points out, low- to mediumrisk women may be better served by midwifery-led care. But expanding midwifery-led care can be challenging for state Medicaid agencies, and Medicaid managed care organizations, coordinating care for Medicaid recipients.

Midwifery-led care can benefit both the patient receiving care and the health care system. The report references a review that found "lower rates of cesarean deliveries, preterm births, and interventions such as labor induction and regional anesthesia, and higher rates of breastfeeding." Overuse of unnecessary medical interventions are expensive, so reducing them can help lower costs. Integrating the use of midwives can also reduce clinical burnout on physicians.

"To ease the burden of developing a midwifery-led care approach, the Institute of Medicaid Innovation's report answers specific, critical questions about variations in access and coverage between the Medicaid population and commercially insured patients, about billing codes and quality measures, and emergency payment and contracting
approaches, among others."

To ease the burden of developing a midwifery-led care approach, the Institute of Medicaid Innovation's report answers specific, critical questions about variations in access and coverage between the Medicaid population and commercially insured patients, about billing codes and quality measures, and emergency payment and contracting approaches, among others. The report also features case studies that identify best practices, innovative initiatives, and lessons learned in making midwifery-led care more available.

The community of Hope, a nationally accredited birth center that is linked to a large Federally Qualified Health Center (FQHC) in Washington D.C., offers an example of an alternative payment model that makes midwifery-led care more accessible. By joining the birth center with an FQHC, the organizations have been able to share fixed costs. Beyond financial savings, though, the part- 
nership has brought midwifery-led care to the community through a trusted organization-the FQHC. Even though most women who get prenatal care at Community of Hope deliver at hospitals, the case study points out, "families continue to visit the clinic for their ongoing health needs after birth."

Baby and Company, a Nashville-based for-profit company, developed as a network of private equity-backed, freestanding birth centers, provides an example of how some Medicaid payment and contracting provisions can impact the profitability of the midwifery-led model. It encountered insurmountable financial and regulatory barriers that forced the Company to close all but one birth center. Baby and Company's experience underscores the importance of statewide policies that increase access to and support the sustainability of freestanding birth centers.

\section{"By leveraging the opportunities identified in the Institute for Medicaid Innovation report, policymakers can help make the business case, increase awareness, and clear a policy path for expanding midwifery-led care."}

Research indicates an opportunity to improve maternal health outcomes through expanded use of the midwifery-led model of care. By leveraging the opportunities identified in the Institute for Medicaid Innovation report, policymakers can help make the business case, increase awareness, and clear a policy path for expanding midwifery-led care.

Reference:

1. https://www.medicaidinnovation.org/ images/content/2020IMI-Improving Maternal Health Access Coverage and Outcomes-Report.pdf

The author has not indicated any disclosures.

NT

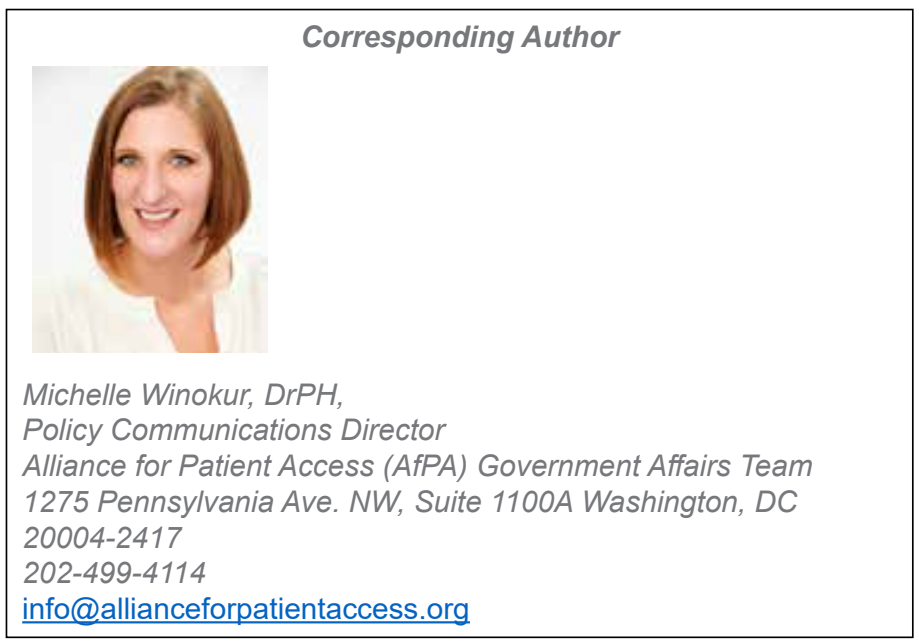

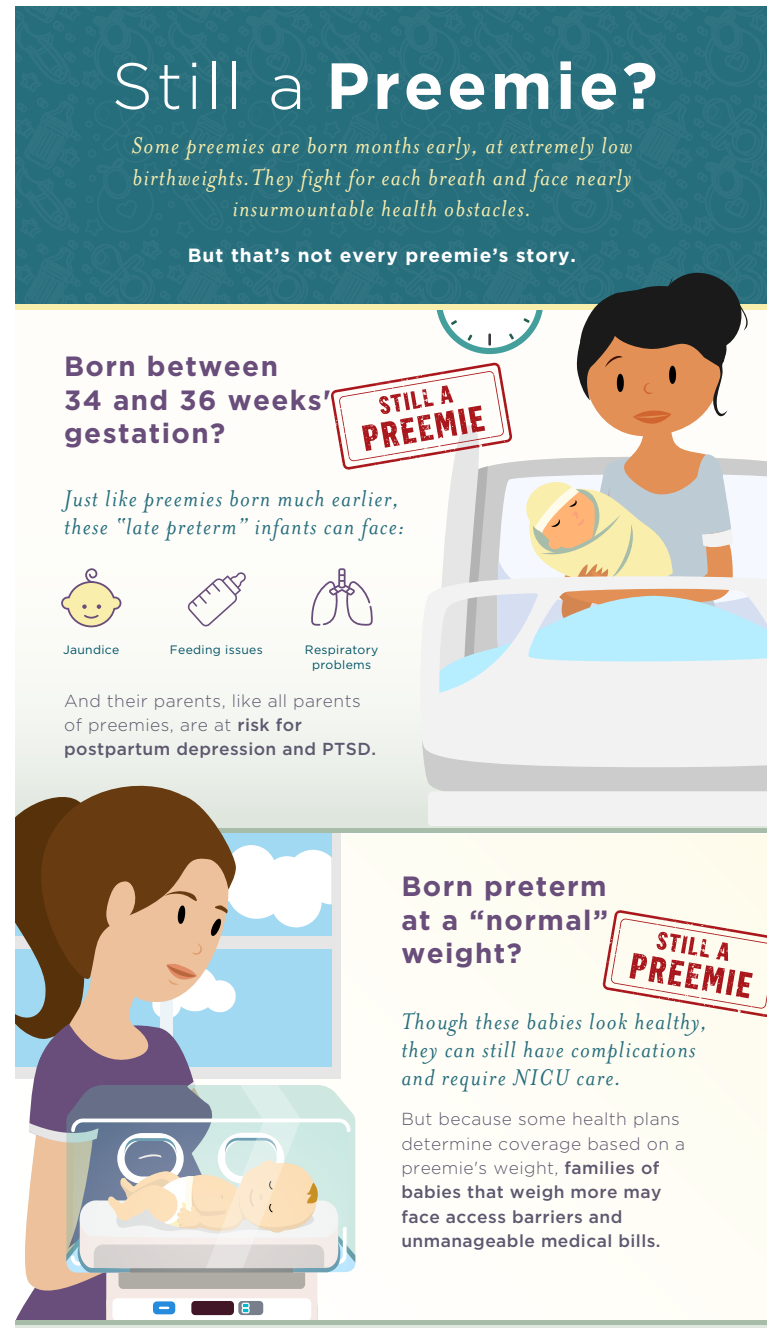

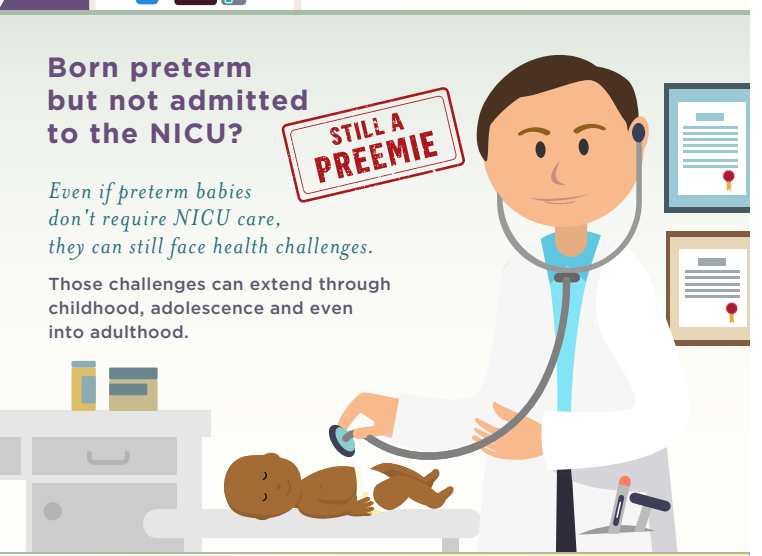

Some Preemies

Will spend weeks

in the hospital

Th Will have lifelong

Qำ Are disadvantaged

Will from birth

$\mathrm{NCfIH}$ 糹ational Coalition health problems 